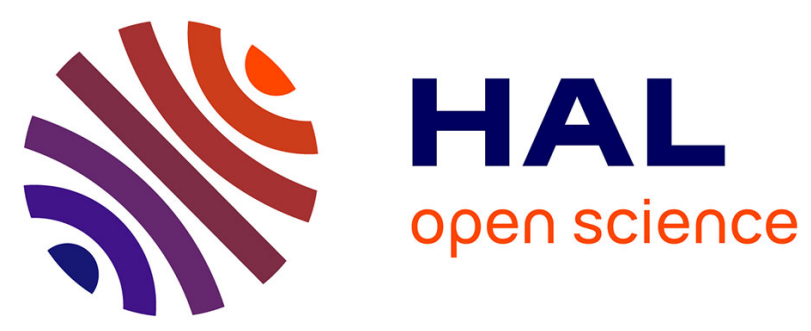

\title{
Combined conceptual and preliminary aerodynamic design of a low-boom Supersonic Civil Transport aircraft for holistic evaluation of its environmental impact
} Gérald Carrier, Jérémy Cadillon, Pierre Grenson, Olivier Atinault, Maxime Huet, Franck Morel, Sébastien Defoort

\section{To cite this version:}

Gérald Carrier, Jérémy Cadillon, Pierre Grenson, Olivier Atinault, Maxime Huet, et al.. Combined conceptual and preliminary aerodynamic design of a low-boom Supersonic Civil Transport aircraft for holistic evaluation of its environmental impact. AIAA AVIATION 2021 FORUM, Aug 2021, VIRTUAL EVENT, United States. 10.2514/6.2021-2568 . hal-03394927

\section{HAL Id: hal-03394927 \\ https://hal.science/hal-03394927}

Submitted on 22 Oct 2021

HAL is a multi-disciplinary open access archive for the deposit and dissemination of scientific research documents, whether they are published or not. The documents may come from teaching and research institutions in France or abroad, or from public or private research centers.
L'archive ouverte pluridisciplinaire HAL, est destinée au dépôt et à la diffusion de documents scientifiques de niveau recherche, publiés ou non, émanant des établissements d'enseignement et de recherche français ou étrangers, des laboratoires publics ou privés. 


\title{
Combined conceptual and preliminary aerodynamic design of a low-boom Supersonic Civil Transport aircraft for holistic evaluation of its environmental impact
}

\author{
Gérald Carrier $^{1}$, Jérémy Cadillon ${ }^{2}$, Pierre Grenson ${ }^{3}$, Olivier Atinault ${ }^{4}$ \\ DAAA, ONERA, Université Paris Saclay \\ F-92190, Meudon-France \\ Maxime Huet ${ }^{6}$ \\ DAAA, ONERA, Université Paris Saclay \\ F-92322, Châtillon - France \\ Franck Morel $^{7}$, Sébastien Defoort ${ }^{8}$ \\ ONERA / DTIS, Université de Toulouse \\ F-31055 Toulouse - France
}

The renewed interest for Supersonic Civil Transport on one side and the environmental challenges that whole aeronautics sector will have to overcome in the next decades calls for an holistic evaluation of the environmental impacts of a possible reintroduction of supersonic flights after Concorde retirement in 2003. In this perspective, a research effort has been initiated by ONERA to investigate the lowest levels of environmental impacts that can be expected by a new generation of civil supersonic aircraft. This paper presents the set-up and verification of different models and of a methodological design approach combining conceptual and preliminary aircraft design capabilities. This approach is intended to design a supersonic civil transport aircraft concepts that can serve to evaluate the minimum levels of environmental impacts of new supersonic aircraft integrating state-of-the-art technologies in terms of sonic boom, take-off noise and emissions.

\footnotetext{
${ }^{1}$ Research Scientist, Aerodynamics, Aeroelasticity and Acoustics Department and AIAA Member.

${ }^{2}$ Research Scientist, Aerodynamics, Aeroelasticity and Acoustics Department.

${ }^{3}$ Research Scientist, Aerodynamics, Aeroelasticity and Acoustics Department.

${ }^{4}$ Research Scientist, Aerodynamics, Aeroelasticity and Acoustics Department.

${ }^{6}$ Research Scientist, Aerodynamics, Aeroelasticity and Acoustics Department.

${ }^{7}$ Research Scientist, Information processing and Systems Department.

${ }^{8}$ Research Scientist, Information processing and Systems Department.
} 


\section{Nomenclature}

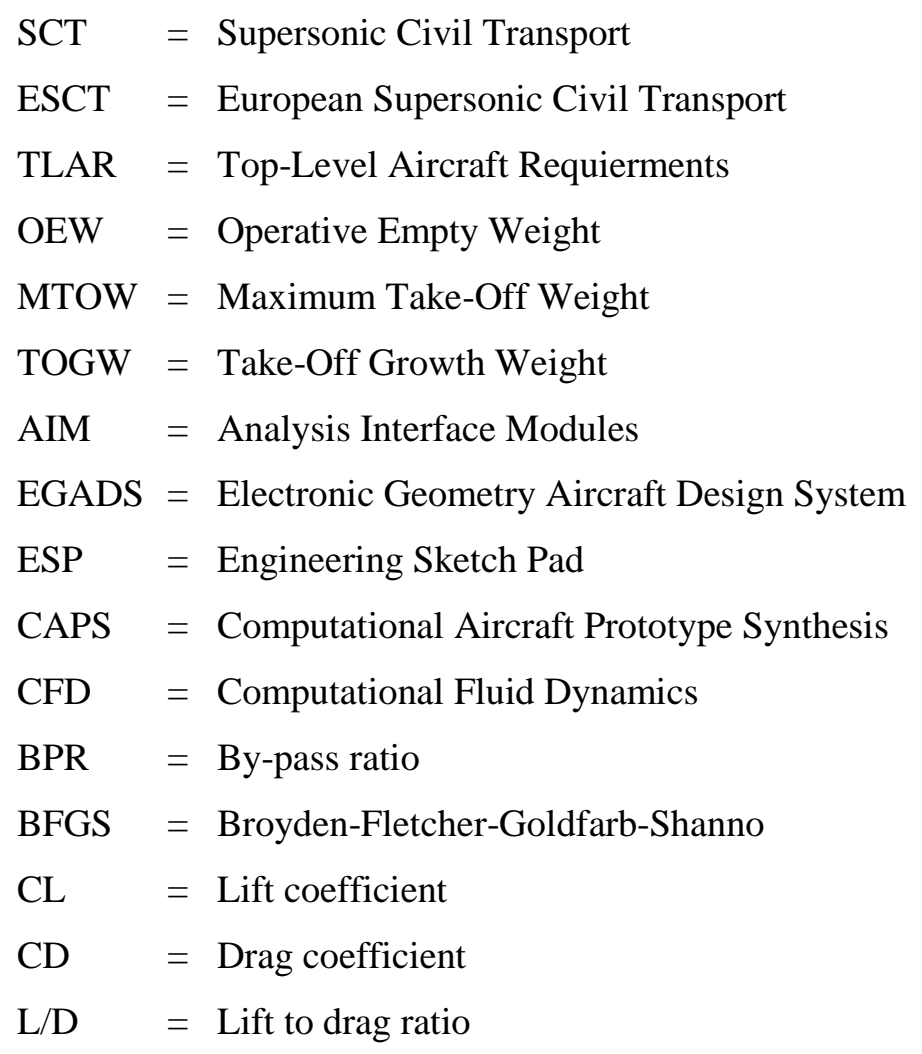

\section{II.Introduction}

After the retirement of Concorde, ending in 2003 the erea of supersonic civil flights opened in the 1970s, a renewed and growing interest for supersonic civil transport has emerged since about 2010 with several companies working on projects of new supersonic civil aircraft concepts and significant research efforts dedicated to supersonic flight technologies in US, Japan and Europe. In this context, ONERA has initiated in 2020 a research effort combining conceptual design with a concomitant preliminary aerodynamic and low-boom design of a Supersonic Civil Transport (SCT) aircraft.

The objective of this initiative is twofold. Firstly, to develop capabilities, models and tools for efficient conceptual-to-preliminary aircraft design of low-boom supersonic civil transport concepts. Secondly, to design the basis of supersonic concept plane with its associated multidisciplinary set of data. Such a research platform could serve ultimately in future design and technological development studies, especially those intended to evaluate the overall environmental impacts of an optimized supersonic transport aircraft concept embarking advanced technologies.

This paper describes the work carried out during the first year of this research which covered the development and verification of both multidisciplinary models needed for conceptual design of SCT and 
high-fidelity aerodynamic models needed for low-boom aeroshape design. The first section describes the set-up and verification of the different low-fidelity multi-disciplinary models necessary for the conceptual design of a supersonic aircraft, together with the retained TLAR for exercicing and verifying these models. The second section describes the models, tools and and design approach proposed to deal with both aerodynamic and sonic boom design. Finally, the third section focuses on the engine integration aspects.

\section{III.Top Level Aircraft Requirements and conceptual design models preparation and verifications}

\section{A. TLAR}

Based on existing market studies[1], recent and ongoing studies in industry and academia[2][3][4][5][6][7][8] (and a large a priori), the following Top Level Aircraft Requirements (TLAR) have been selected for this design studies:

- a range of $5000 \mathrm{Nm}$,

- a payload $16.000 \mathrm{lb}(\sim 80 \mathrm{pax})$,

- a low-boom cruise at a design Mach number of 1.6.

\section{B. Multidisciplinary model calibration}

In order to perform the conceptual design of a supersonic aircraft concept fulfilling the TLAR defined before, disciplinary models specific to supersonic aircraft providing realistic data are needed, especially for aerodynamic performance and mass estimation. The first work carried out has been to identify and select the available models at ONERA for supersonic transport aircraft evaluation and verify the response of those models by comparing their output against existing data in literature for supersonic aircraft. In these verification exercise, the data for Concorde and the Lockheed-Martin LM1044 concept[6] have been used.

\section{Weight/Structure}

At the conceptual design level of a project, statistical models are often used in order to evaluate the weight of the aircraft. Due to a poor statistical sample of commercial supersonic aircraft, such an approach is impossible to use. A structural approach is more appropriate for a brand new configuration but is complex, need long developments and generate unsuitable computation times at the conceptual level. That's why a derived method was first used, i.e. an empirical method based on the mass of a known aircraft, Concorde. The empty weight of the aircraft is divided into several elementary component and the mass of each component is a function of relative parameters (the ratio of the parameter of the new configuration and Concorde's parameter) [12].

This model has been evaluated on different configurations of old and new generation of civil transport aircraft. To illustrate the results obtained, we first evaluated the OEW of the ESCT (European Supersonic 
Civil Transport) which was a project of future supersonic transport aircraft in the end of the 90's. It was a 3 classes aircraft, carrying 252 passengers at $5500 \mathrm{Nm}$ with a supersonic cruise at Mach 2.05.

\begin{tabular}{|l|c|c|c|}
\hline & ESCT & Derived method & Difference \\
\hline Structure (kg) & 74595 & 95064 & $+27.44 \%$ \\
\hline Propulsion (kg) & 33825 & 32580 & $-2.7 \%$ \\
\hline Systems \& Operating items (kg) & 33942 & 29881 & $-12 \%$ \\
\hline OEW (kg) & 142362 & 157525 & $+10.65 \%$ \\
\hline Payload (kg) & 24000 & 24000 & \\
\hline Fuel (kg) & 176800 & 176800 & \\
\hline TOGW (kg) & 343162 & 358325 & $+4.4 \%$ \\
\hline
\end{tabular}

Figure 1 - Comparison of ESCT weight statement

Compared with weight estimation of the ESCT, the derived model seems to overestimate the structural weight $(+27.44 \%)$ partially compensated by an underestimation of the systems ans operating items $(-12 \%)$. Only the propulsion weight is estimated with a good accuracy (Figure 1). The overall accuracy of the OEW is acceptable at the conceptual design stage $(+10.65 \%)$, this difference representing only $4.4 \%$ on the TOGW.

In order to illustrate new generations of supersonic transport aircraft, the second evaluation is based on a the LM1044 configuration. With this configuration, the accuracy of the structural weight estimation is excellent $(-0.7 \%)$ (Figure 2$)$, the systems and operating items weight estimation is good $(+8.2 \%)$ and only the propulsion weight overestimated $(+31.6 \%)$. This can be explained by an important difference in technology between the engines of Concorde and the LM1044. Finally, the difference between the published LM1044 OEW and the computed OEW with the derived method represents only $4600 \mathrm{~kg}$ (or $7.2 \%$ ) and only $3.2 \%$ if we consider the TOGW. 


\begin{tabular}{|l|c|c|c|}
\hline & LM 1044 & Derived method & Difference \\
\hline Structure (kg) & 33714 & 33473 & $-0.7 \%$ \\
\hline Propulsion (kg) & 14169 & 18644 & $+31.6 \%$ \\
\hline Systems \& Operating items (kg) & 15449 & 16711 & $+8.2 \%$ \\
\hline OEW (kg) & 64232 & 68828 & $+7.2 \%$ \\
\hline Payload (kg) & 7257 & 7257 & \\
\hline Fuel (kg) & 73736 & 73736 & \\
\hline TOGW (kg) & 145225 & 149821 & $+3.2 \%$ \\
\hline
\end{tabular}

Figure 2: Comparison of LM1044 weight statement

To conclude, the derived method provides TOGW estimations with an appropriate accuracy for conceptual design studies.

\section{Aerodynamics}

Several aerodynamic models are available, depending on the phase of flight studied:

- A model based on the DATCOM method[13] for subsonic phases $(0 \leq$ Mach $\leq 0.95)$. This model computes balanced polar and can evaluate the effect of high lift devices.

- A model based on the slender body theory for supersonic phases (Mach $\geq 1.7)$

- An empirical model based on RAYMER publications[14] for the other phases $(0.95<\mathrm{Mach}<1.7)$

These three models, adapted to cranked wing and tubular fuselage configurations, are tested on the LM1044 configuration. The benchmark results (Figure 3) are from [6] and the geometric datas required for calculation result from measurements taken on the different plans provided in the same report.

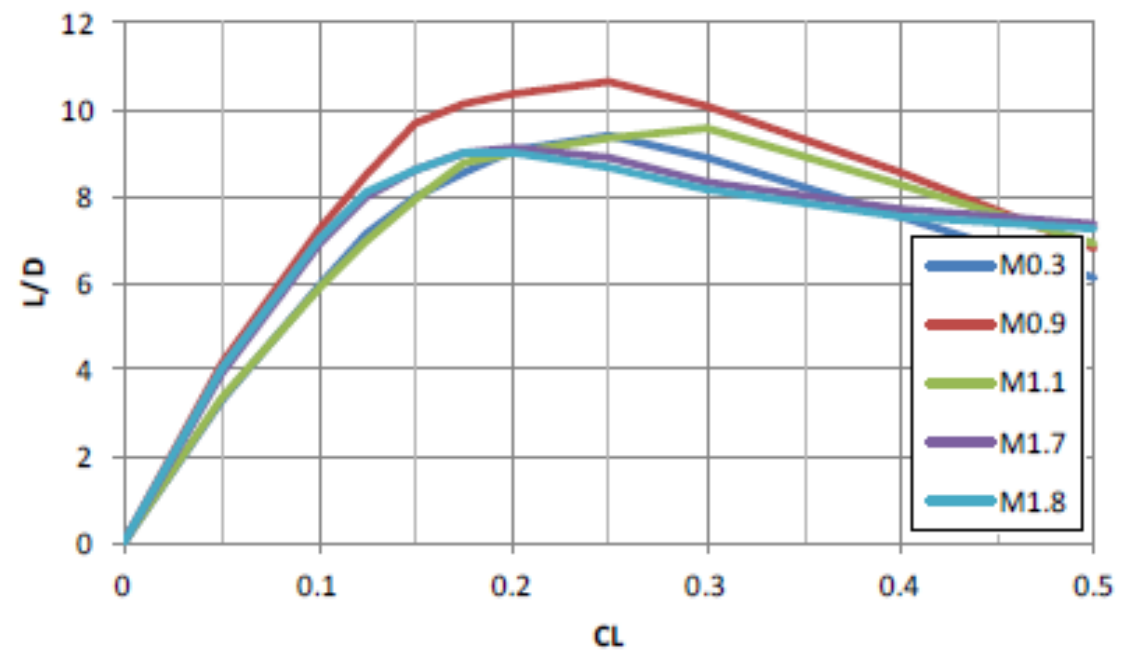

Figure 3: Reference LM 1044 L/D ratio 
At subsonic speeds, the calculated maximum fineness is slightly lower than the published maximum fineness and are obtained at lower CL. For example, the (L/D)max at Mach 0.3 is equal to 8.4 and is obtained at CL equal to 0.16 whereas the published results show a (L/D)max equal to 9.5 at $\mathrm{CL}$ equal to 0.25 . For all Mach numbers, we can notice a discontinuity in the fineness between 0.17 and 0.22 due to a slope change in the evolution of $\operatorname{CL}(\alpha)$.

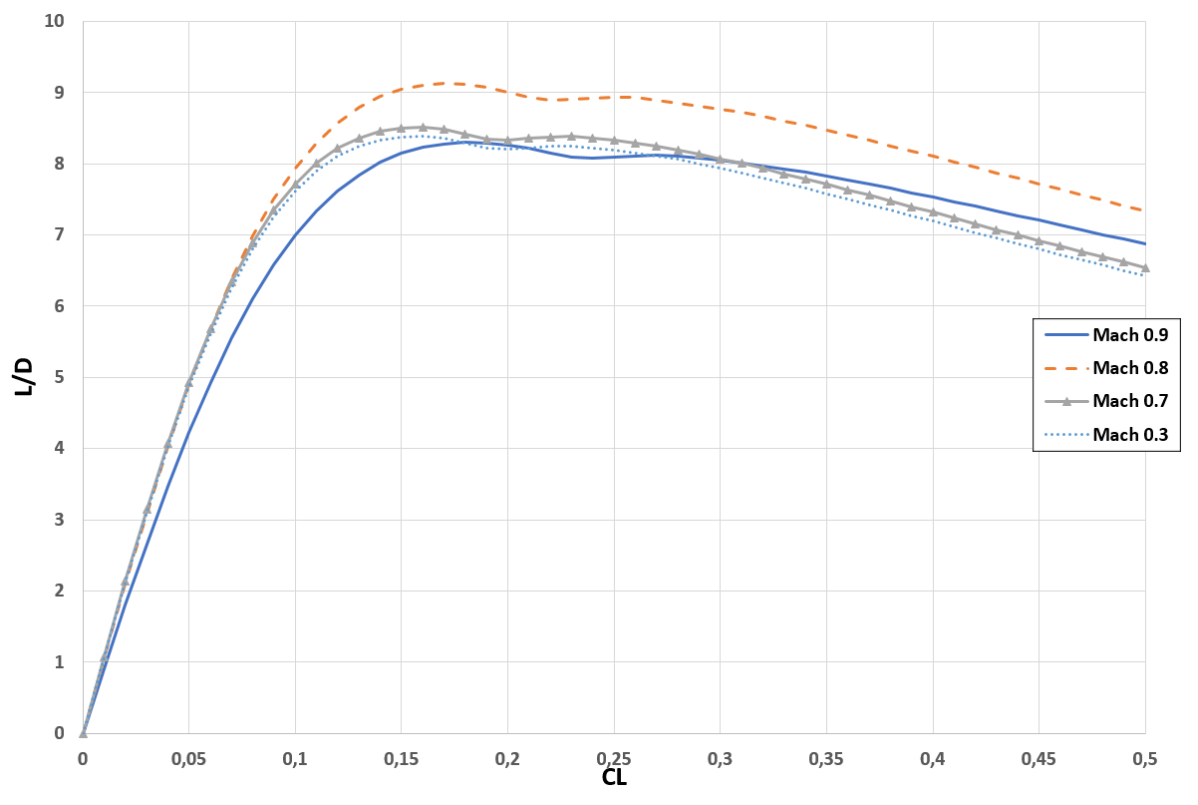

Figure 4 - Subsonic speed LM1044 L/D ratio

At supersonic speeds, the calculated (L/D)max is comparable to that published (about 9.5) but are also obtained at lower CL (between 0.13 and 0.14 against 0.17 and 0.18 ). A quick calculation shows that at the altitude of $60000 \mathrm{ft}$ with an estimated mass of $119000 \mathrm{~kg}$ at the beginning of a Mach 1.7 cruise, the flight CL is equal to 0.18 . In these conditions, the calculated L/D by the aerodynamic model is equal to 9.3, i.e. a value very similar to that published. 


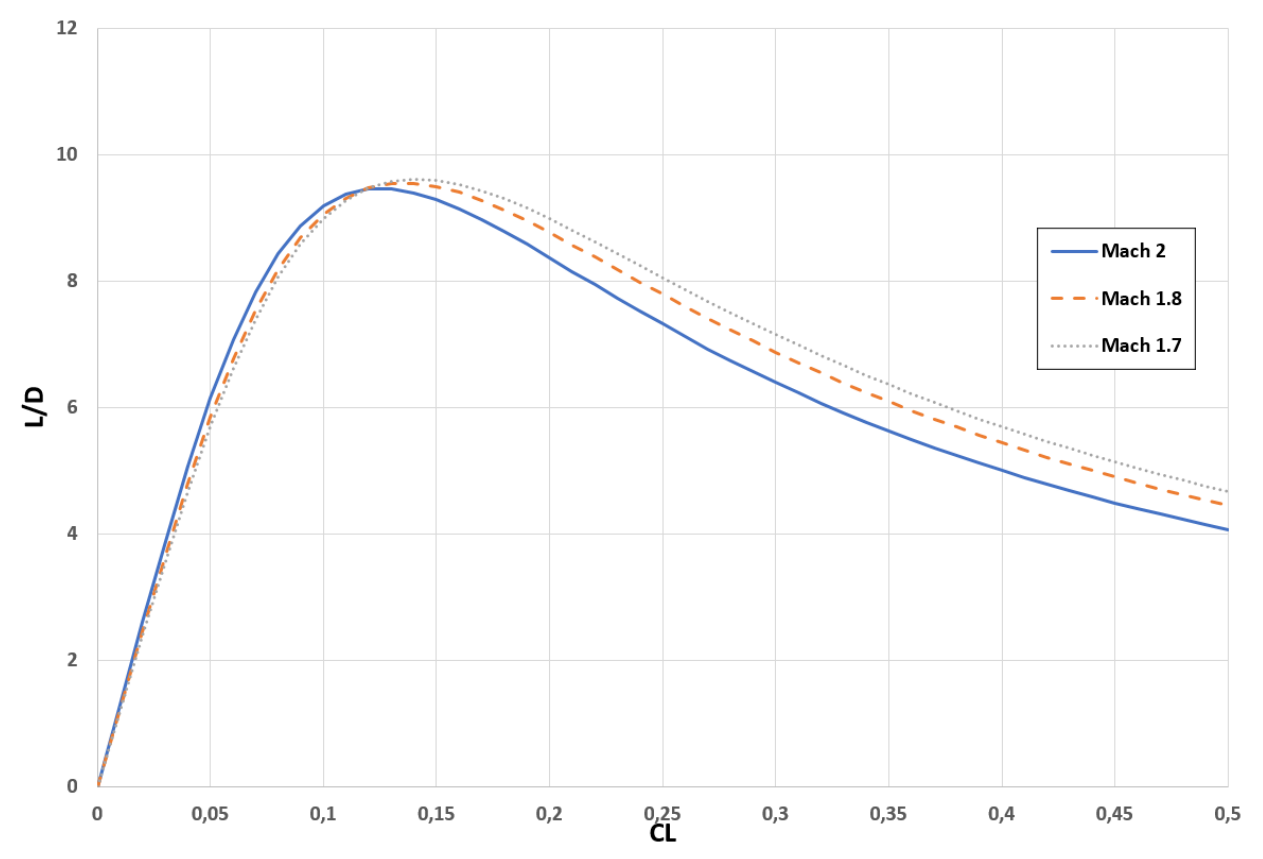

Figure 5 - Supersonic speed LM1044 L/D ratio

\section{Development of a first ONERA low-boom supersonic aircraft concept}

To support the set-up and verification of the multi-disciplinary models and exercice the proposed multilevel design approach a first concept plane has been defined, named ONEX0 (Figure 6) for the TLAR introduced in section III-A.

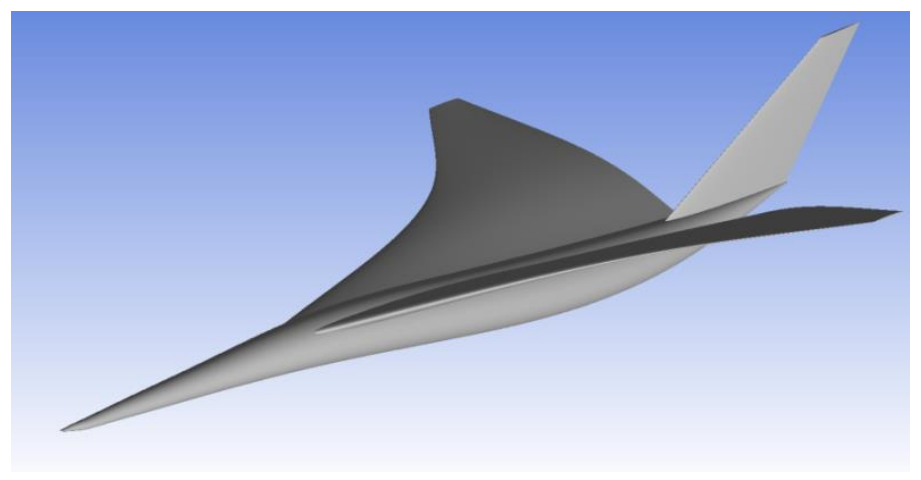

Figure 6: OML of initial design for the conceptual design (ONEX0 configuration)

\section{IV.Models and tools for aerodynamic and low boom design}

A. Development of a parametric automated aerodynamic and sonic boom evaluation and design environment

For the sake of performing the aerodynamic design and low-boom shaping of the ONEX0 configuration, the following design framework has been set up (Figure 7). First, the geometry has been generated using 
the Engineering Sketch Pad (ESP)[9] tool developed by MIT. This freely available and open source software allows the user to build its own geometry with different levels of fidelity. This geometry can be connected to Analysis Interface Modules (AIM) through the Computational Aircraft Prototype Synthesis (CAPS)[11] software (for meshing, structural analysis,..). In the current framework, four AIM modules are used and assembled into an automated design analysis chain using plain Python scripts (Figure 7):

1. Pointwise (meshing),

2. SU2 (CFD calculations),

3. FRICTION, a viscous drag evaluation module relying on analytical laminar/turbulent skin friction formula,

4. and a in-house Sonic Boom evaluation module.

The commercial mesh generation software Pointwise enables the engineer to create structured and unstructured meshes, covering a wide range of CFD calculation needs. It is used there in batch mode using the Glyph scripts capability that enables to automate the generation of unstructured CFD mesh around almost arbitrary aircraft geometries[15]. Then, the open-source CFD software SU2 [17] written in C++ for the numerical solution of partial differential equations, provides the flow field around a geometry and thus, the aerodynamic coefficients (solving either the RANS or Euler equations).

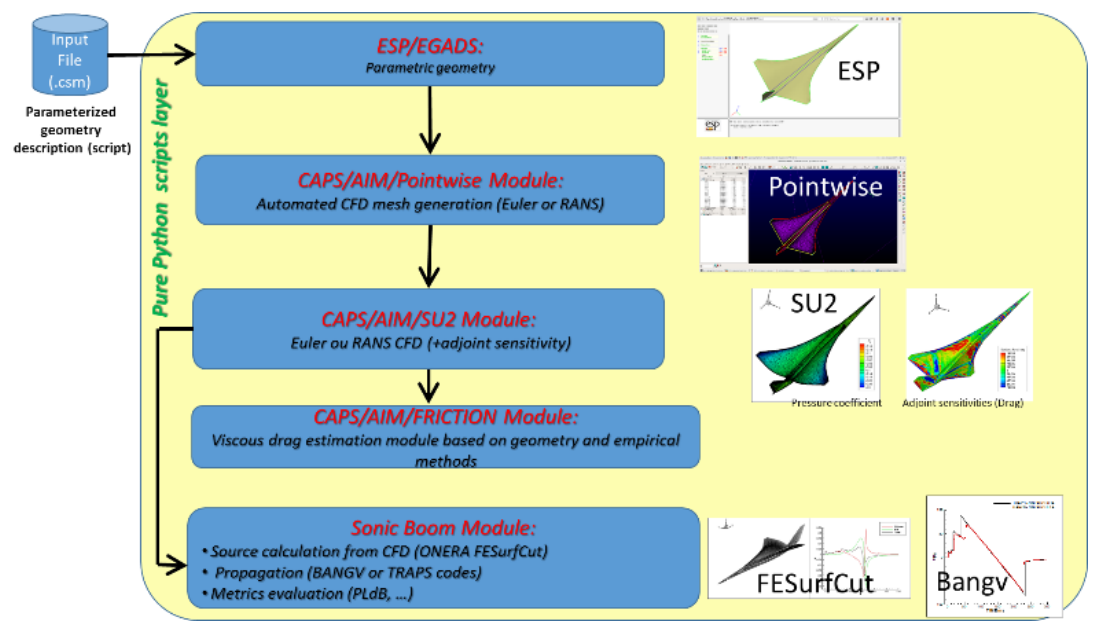

Figure 7 : Aerodynamic/Sonic Boom design framework based on CAPS/ESP/EGADS tools

In the case where Euler CFD solutions are calculated, the total drag of the aircraft is evaluated by adding the viscous drag component evaluated with the FRICTION module with the wave and induced drag components extracted from the CFD results.

The typical turn-around times of the different modules composing the full process described in Figure 7 are: 
- Geometry assembly from ESP parametric geometry scripts: few seconds to 1-2 minutes (depending on the geometry complexity: number of geometry components, number of geometrical operations such as union, intersection, ...);

- Meshing: from 3-4 minutes for Euler grid generation of less than 1 million cells to about 30 minutes for RANS meshes of few tens of million cells;

- CFD: typically 1-3 minutes for Euler simulations (on 112 CPUs Intel Xeon « Broadwell » E52680v4) and 30-60 min for RANS simulations (on 448 CPUs);

- FRICTION module: roughly 1 second;

- Sonic boom module: roughly 1 minutes (for one single flight condition, undertack sonic boom evaluation).

\section{B. Sonic boom evaluation module}

For a given aircraft geometry and flight condition, the sonic boom signal on ground and associated sonic boom metric (Perceived Loudness, A-, B-, C- or D-weighted Sound Equivalent Loudness) can be calculated by extracting from the CFD solution in cruise conditions the near-field pressure signature on a cylinder surrounding the aircraft, aligned with the free-stream, with a radius of few aircraft body lengths. This nearfield signature can then be propagated through the atmosphere with a ray-tracing method down to the ground level to produce the pressure-time signal at ground from which sonic-boom loudness metrics can be calculated. Such approaches have been widely validated both for the near-field prediction capability and the far-field atmospheric propagation part [20][21].

Such an approach can be qualified of high fidelity sonic boom prediction method since it requires important CFD efforts to produce high resolution and solution-adapted grids[20] to capture and propagate in the CFD domain all details of the signature up to the extraction distance (typically 3 to 5 aircraft body lengths).

The present approach for sonic boom near-field characterization largely relaxes the requirements put on the CFD grid characteristics by calculating the Whitham function[22] that characterizes the aircraft sonic boom source directly from the CFD pressure solution on the aircraft skin and not in the volume (saving the efforts and CFD calculation time associated to a dense solution-adapted grid in the volume). Such an approach can be qualified of medium fidelity in the sense that it relies on the Whitham corrected supersonic linearized theory [22] and its extension to lifting bodies proposed by Walkden [23].

Such an approach proceeds by calculating, for a given azimuth angle, an equivalent area distribution from the geometry of the aircraft and the attached pressure field (extracted from the CFD solution). This area is the sum of two components: a volume contribution and a contribution due to the lift. Both are 
calculated by slicing the geometry according to planes tangent to the Mach cone with normal defined by the azimuth angle of interest, as illustrated in Figure 8 and detailed in [5].

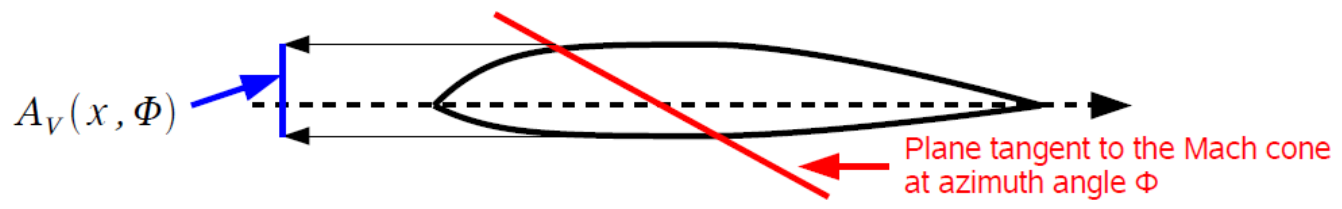

Figure 8 : Illustration of the cutting plane (in red) used to calculate volume contribution $A_{v}$ to the equivalent area $A_{e}$ at a given location $x$ and for a given azimuth angle $\Phi$.

For a given azimuth angle at which one wants to propagate the sonic boom and from the $\mathrm{A}_{\mathrm{e}}(\mathrm{x})$ distribution, the equivalent Whitham function can be calculated from:

$$
F(\xi, \Phi)=\frac{1}{2 \pi} \int_{0}^{\xi} \frac{\frac{\partial^{2} A e}{\partial t^{2}(t, \Phi)}}{\sqrt{\xi-t}} d t \quad \text { with: } A_{e}(x, \Phi)=A_{V}(x, \Phi)+A_{\text {lift }}(x, \Phi)
$$

From which the near-field pressure perturbation can be determined at a given distance $r$ from the fuselage axis by:

$$
\frac{\Delta P}{P_{0}}(x, r, \Phi)=\frac{\gamma M_{0}{ }^{2}}{\sqrt{2 \beta r}} F(\xi, \Phi), \quad \text { with }: x=\xi+\beta r-\frac{(\gamma+1) M_{0}^{4}}{\sqrt{2 \beta^{3}}} \sqrt{r} F(\xi, \Phi) \text { and } \beta=\sqrt{1-M_{0}{ }^{2}}
$$
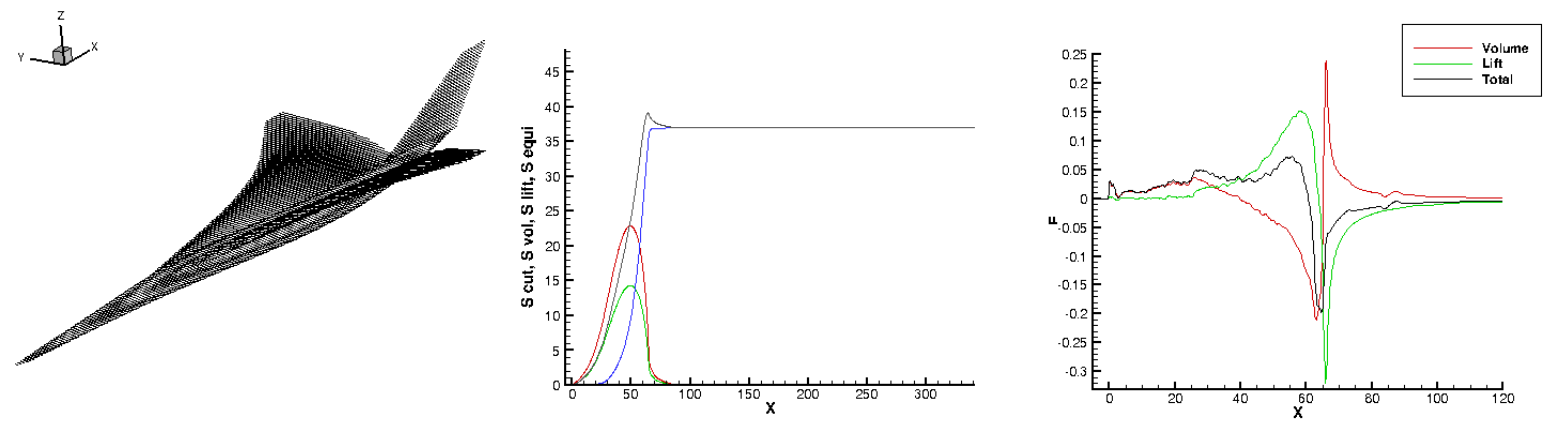

Figure 9 : Example of the application of ONERA SurfCut code to extract Whitham function from surface CFD solution. Multiple slices of the geometry (left), Equivalent distributions build-up from lift and volume contributions (center), resulting Whitham F-functions (right).

This method has been implemented at ONERA in a in-house code, SurfCut, which takes as input the surface CFD solution on the aircraft skin. Typical results of this approach are illustrated in Figure 9. This code, in combination with a ray tracing code such as TRAPS[25] or BANGV[26] is able to provide an evaluation of the ground propagated sonic boom of sufficient quality for the sake of low-boom preliminary and intermediate design, as shown in Figure 10. 


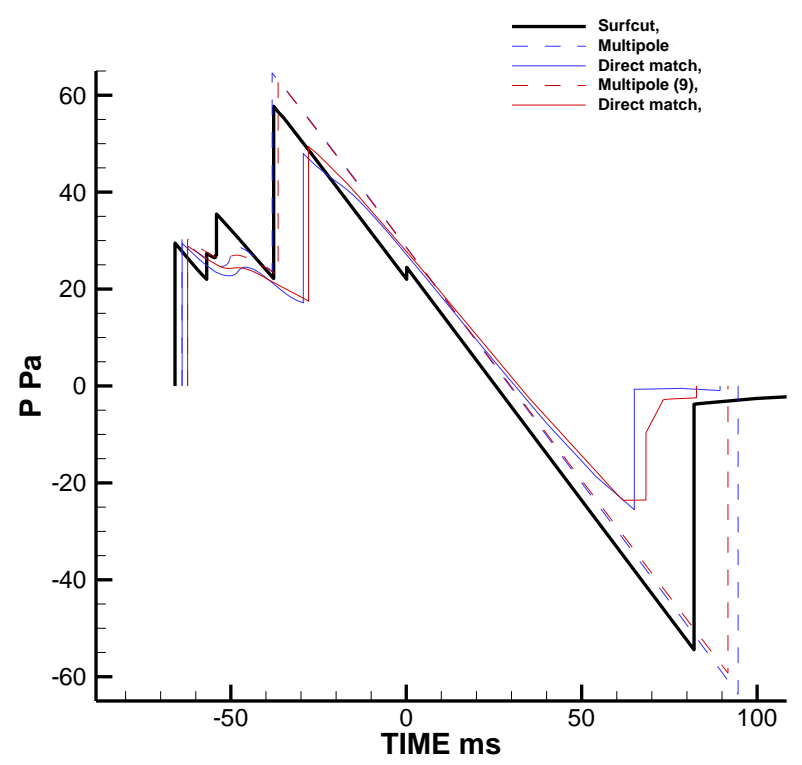

Figure 10 : Comparison of the ground propagated sonic boom signal evaluated from a near-field pressure signature evaluated from the SurfCut or a volume extraction in the CFD solution on a cylinder (without and with multipole decomposition[24]).

\section{First applications of the design framework}

As an illustration of the applicability and capability of the tools and framework introduced in section IV.A, two examples of its applications within an automated design optimization process are described: first a fuselage area-rule shaping exercise (Figure 11) and second a wing design optimization (Figure 12).

The first optimization consisted in minimizing drag by varying the diameter of different circular sections along the fuselage. The location of these seven cross sections is shown in Figure 11. The SciPy optimization Nelder-Mead algorithm has been used to find the optimum in the design space of these seven parameters. This region is bounded to avoid excessively small diameters (each diameter lies between 1 meter and 5 meters). Moreover, the upper line of the fuselage is held fixed and thus, only the lower line will change while the optimizer is running (previously, this upper line has been changed compared to the initial geometry).

To reduce the computational cost, an unstructured-mesh Euler method has been chosen. This mesh is made up of 1.7 million cells, allowing for a computation time less than 10 minutes for each evaluated geometry. Each SU2 calculation is performed using the fixed (target) CL constant mode (CL $=0.11)$.

As shown in Figure 11, the different parameters vary in the design region during the first iterations until stabilization is reached after 300 studied geometries. The objective function (essentially pressure drag) decreases. After only 100 iterations, the drag coefficient (based on the reference area: $422.1 \mathrm{~m}^{2}$ ) is equal to 
58.3 drag counts, corresponding to a 63.4 drag count reduction compared with the initial geometry. The Figure 11 depicts the decrease of the front surface by reducing the diameter of the cross sections.

A second optimization exercise has been performed in order to evaluate and assess the capabilities of the assembled tools to help the design of the wing from a pure aerodynamic performance perspective. In this purpose, a wing parameterization has been set up in order to enable the modification of the wing twist and camber (three parameter per section) in three pilot sections, namely inner wing, mid wing and tip wing. Wing planform and thickness distribution are kept constant. Adding the angle of attack to the geometry design variables resulted in a total number of variable of 13 that were optimized to maximize the aerodynamic efficiency using a BFGS gradient method with finite difference for the calculation of sensitivities. Results of this optimization and shown in Figure 12.

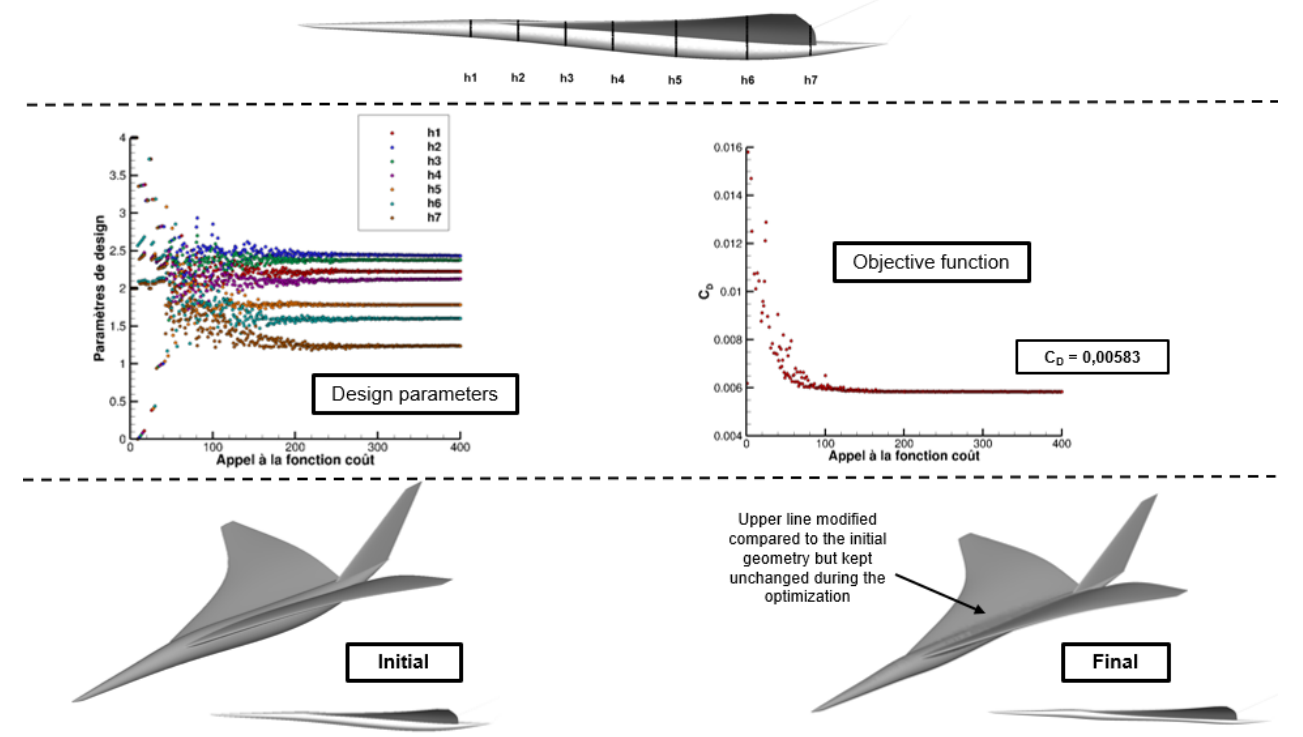

Figure 11: First assessment of fuselage optimization capability 

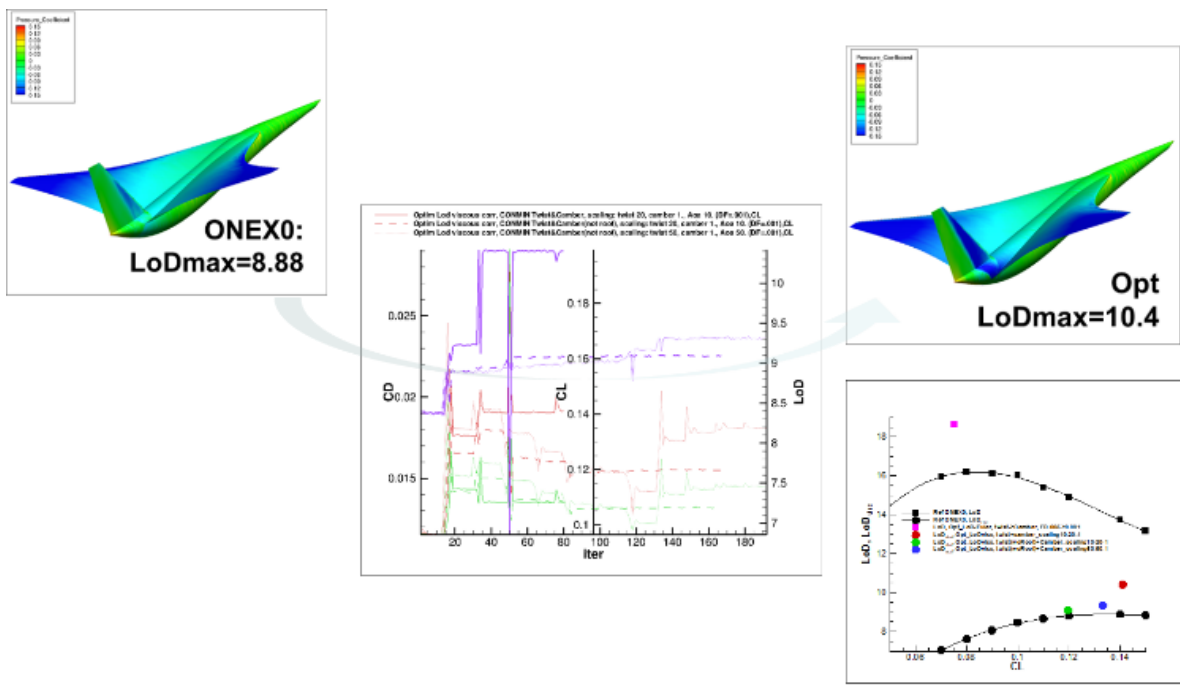

Séminaire DAAÁ -Paramétrisation géométrique pour conception aérodynamique et structure,

\section{.}

\section{Figure 12: First assessment of wing optimization capability}

\section{V.Models \& tools for propulsion system integration}

\section{A. First considerations regarding propulsion system definition}

In order to achieve certifiable LTO noise level, the choice of a medium by-pass-ratio engine technology is considered in this work (3 to 4). Based on a first evaluation of the start of cruise weight and achievable aerodynamic efficiency in terms of lift-to-drag ratio, the thrust requirement in cruise has been estimated. A first sizing of the engine propulsive system has then been performed on the basis of an estimated specific thrust from available data of the Aerion AS2/GE Affinity tandem (Table 1). From the obtained fan inlet diameter, which is close to the diameter of a circular supersonic air intake for a cruise Mach number of 1.6, several engine integration solutions with three or four engines have been considered. The required size of the inlet face of a medium BPR engine makes the integration of the propulsive system with the wingfuselage assembly an important design step to focus on in order to ensure the low-boom/low-drag capabilities of the aircraft in cruise condition. 


\begin{tabular}{|c|c|c|}
\hline & Aerion AS2 & Onera concept plane \\
\hline Engine & GE Affinity $(\mathrm{BPR}=3: 1)$ & Medium-BPR (3:1 or $4: 1)$ \\
\hline Cruise Mach number & 1.4 & 1.6 \\
\hline Fan inlet diameter (m) & 1.33 & 2.09 (3 eng.) / 1.81 (4 eng.) \\
\hline Engine number & 3 & 3 or 4 \\
\hline Total captured area $\left(\mathrm{m}^{2}\right)$ & 4.17 & $\sim 10$ \\
\hline Total mass-flow rate $(\mathrm{kg} / \mathrm{s})$ & 336 & 889 \\
\hline Cruise thrust per engine (lb) & 3500 (engine data) & 10000 (3 eng.) / 7500 (4 eng.) \\
\hline Total cruise thrust (lb) & $\sim 10500$ & $\sim 30000$ \\
\hline Start of cruise weight (lb) & 111000 & 295000 \\
\hline Lift-to-drag ratio & 9.5 (estim.) & 10 \\
\hline Specific thrust (lb/kg/s) & 33 (estim) & 33 \\
\hline
\end{tabular}

Table 1: Inlet captured area estimation based on available data of Aerion AS2 aircraft and GE Affinity engine. Supersonic air intakes are assumed in critical regime in cruise conditions with ingested mass flow rate corresponding to the captured area mass flow.

In a second step, a model for the propulsive system will be derived, which needs to integrate both the medium BPR-engine and the supersonic air inlet. The pyCycle Python library [18] has been selected for this purpose principally because of its high modularity, which allows for adding custom elements to an existing model, and its capability to calculate off-design conditions by numerically solving the non-linear problems associated with each components of the system. This model will be notably employed for evaluation of the required mass flow ratio for the different flight regimes (cruise, landing/take-off, transonic) which are associated with different altitudes, speeds and thrust requirements. These data are necessary to determine the flow regime of the supersonic air intake (subcritical, supercritical) as well as the state of the flow (velocity, temperature) exiting the engine. This state will serve as a boundary condition for the CFD calculations in order to take the engine jet flow into account when assessing the sonic boom in cruise condition and the LTO noise.
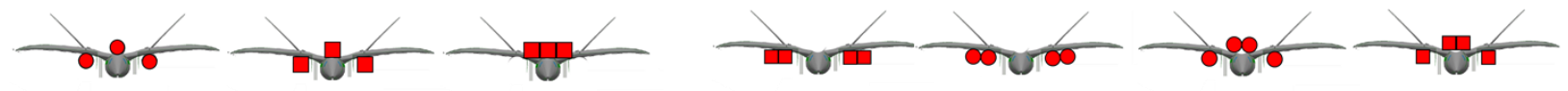

Figure 13: Space of possible propulsive system integration solutions

\section{B. Jet noise estimation}

Urban densification around airports together with the continuous increase of air traffic expose a large number of people to discomfort, in particular noise. This community noise is an important criterion that 
needs to be addressed from the first steps of the conception of supersonic civil transport aircraft if one wants to ensure their acceptation by the people living nearby airports. A precise evaluation of aircraft noise would require its estimation for certification points, that is to say for instance the modelling of all the aircraft noise sources during take-off. Such an evaluation is however time-consuming and irrelevant in the early stages of conception, and simpler and faster solutions are better suited.

The following assumptions are retained here. First, engine noise is considered to be the dominant noise source during take-off. Due to the medium by-pass ratio of the engine, fan noise is neglected and only jet noise is accounted for. Second, noise radiation is not evaluated during the complete take-off phase, but on a single engine condition relevant of take-off. It is believed this is sufficient to converge towards a lownoise engine configuration (through an optimization process, for instance): any reduction or increase of noise for this condition would qualitatively lead to similar variations for other engine conditions.

Jet noise estimation is provided by the model of Stone et al [19] which is well-suited for the evaluation of single- and double-stream jet noise for low to moderately high by-pass ratio engines. The model provides far-field acoustic spectra in third-octave bands for any observer position. It considers isoled jets, so that acoustic shielding by the fuselage and jet-jet interaction noise, for instance, are not included. This model may appear a little bit crude but is sufficient for preliminary design. Noise predictions are moreover very fast, which makes the model pertinent for its integration in an optimization process.

For given engine exhaust geometry and flow conditions (fluid velocity and temperature), the model can provide ground-noise estimations and help discriminating between quiet and noisy configurations. This is for instance the case when comparing three- and four-engine configurations or engines with similar thrust but different geometries and exhaust flows. It may also be used in the aim of low-noise engine design: it provides an acoustic criterion that can be included in an aerodynamic-acoustic optimization process of the propulsion system. From the acoustic point of view, the objective chosen is the reduction of the acoustic power of the engine. It is evaluated from the modelled pressure spectra and can include specific weighting (e.g. A weighting) to take into account the sensitivity of human ear.

\section{First 3D model of engine nacelles and engine integration studies}

Different options for the propulsive system integration have been investigated, mostly a three or four engines configuration with either axisymmetric or 2D (rectangular) inlets have been envisaged and considered and three dimensional geometrical models for these two types of nacelle/inlet (through-flow nacelles) have been build-up with ESP/EGADS (Figure 14). The nacelles have been sized to achieve a mass flow compatible with the estimated thrust requirement in cruise condition. CFD calculation of the isolated nacelle where conducted to perform a first design on the inlet compression system. In a second stage, the 
axisymmetric nacelle configuration has been integrated to the airframe. The complete configuration has been evaluated using CFD in order to:

- Refine/adapt the design of the internal inlet compression system for efficient inlet operation on the aircraft;

- Evaluate the engine installation drag and start optimizing the positioning of the propulsive system (Figure 15).
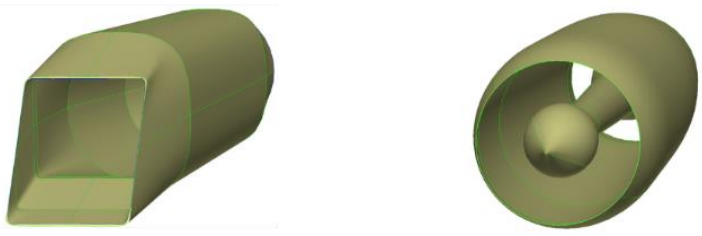

Figure 14: OML of considered engine nacelles solution modelled with ESP/CAPS

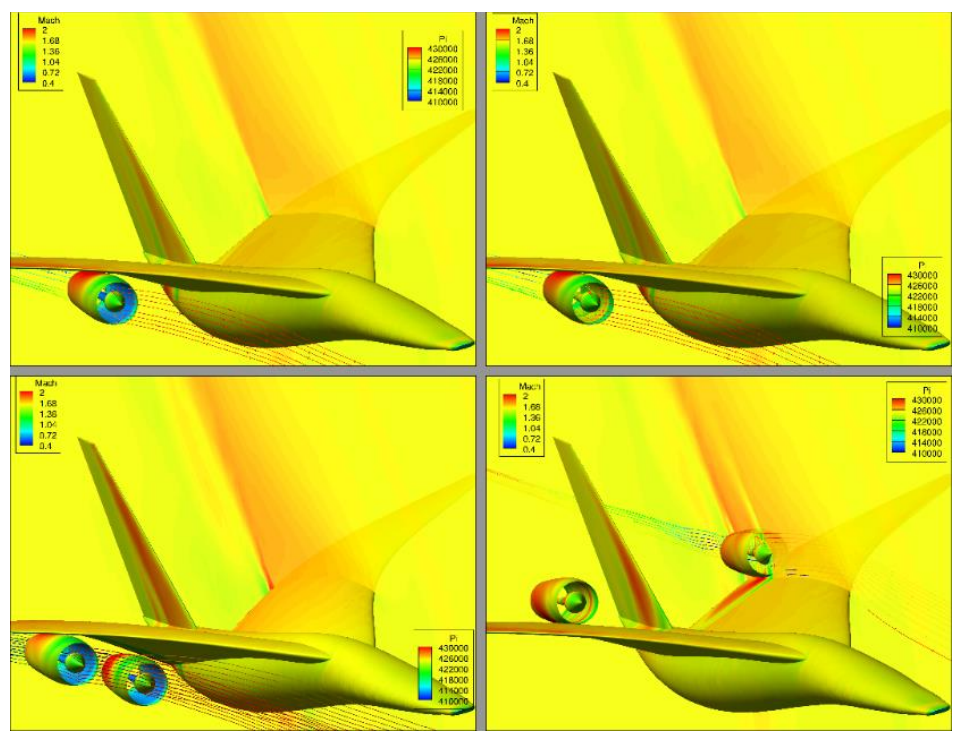

Figure 15: Investigation of different engine integration solution and resulting engine integration drag and inlet performance by mean of Euler CFD

\section{VI.Conclusion and future work}

In 2020, a framework of tools combining conceptual exploration and preliminary aerodynamic design for low-boom supersonic civil transport aircraft has been set up by ONERA and the first design loops based on this framework have been initiated. This design activity will be continued in 2021 with the objective of producing SCT research platforms for future detailed analysis of environmental impacts of possible future 
supersonic civil aircraft. In this perspective, take-off noise (jet noise) considerations will be integrated in the future design cycles.

\section{VII.Acknowledgments}

The authors are grateful to Ingrid LeGriffon from ONERA for fruitful discussions about jet noise modeling.

\section{VIII.References}

[1] Bernd Liebhardt, Klaus Luetjens, Volker Gollnick, "Estimation of the Market Potential for Supersonic Airliners via Analysis of the Global Premium Ticket Market", Conference Paper, DOI: 10.2514/6.2011-6806

[2] Rallabhandi, S., Mavris, D., "Aircraft Geometry Design and Optimization for Sonic Boom Reduction”, AIAA Journal of Aircraft, Vol. 44, No. 1, pp. 35-47, 2007ONERA CISAP

[3] G. Carrier, "Multi-disciplinary optimization of a supersonic transport aircraft wing planform", Paper presented at ECCOMAS 2004 conference, Jyväskylä (Finlande), 24-28 July 2004

[4] J. Brezillon, G. Carrier, M. Laban, "Multidisciplinary Optimization of Supersonic Aircraft Including Low-Boom Considerations", October 2011, Journal of Mechanical Design 133(10), DOI: 10.1115/1.4004972

[5] A. Minelli, I. Salah El Din, G. Carrier, "Inverse Design Approach for Low-Boom Supersonic Configurations", October 2014, AIAA Journal 52(10):2198-2212, DOI: 10.2514/1.J052834

[6] John Morgenstern, Michael Buonanno, Jixian Yao, Mugam Murugappan, Umesh Paliath, Lawrence Cheung, Ivan Malcevic, Kishore Ramakrishnan, Nikolai Pastouchenko and Trevor Wood, Steve Martens, Phil Viars, Trevor Tersmette, Jason Lee, Ron Simmons, and David Plybon, Juan Alonso, Francisco Palacios, Trent Lukaczyk, and Gerald Carrier, “Advanced Concept Studies for Supersonic Commercial Transports Entering Service in the 2018-2020 Period Phase 2", NASA technical report NASA/CR—2015-218719

[7] Harry R. Welge, John Bonet, Todd Magee, Chet Nelson, Eric Adamson, Steve Baughcum, Robert T. Britt, Gregory Miller, Jimmy Tai, "N+2 Supersonic Concept Development and Systems Integration", NASA technical report NASA/CR-2010-216842

[8] Jeffrey J. Berton, Dennis L. Huff, Jonathan A. Seidel, Karl A. Geiselhart, “Supersonic Technology Concept Aeroplanes for Environmental Studies”, AIAA Paper 10.2514/6.2020-0263 
[9] R. Haimes and J. F. Dannenhoffer, "The Engineering Sketch Pad: A Solid-Modeling, FeatureBased, Web-Enabled System for Building Parametric Geometry," in 21st AIAA Computational Fluid Dynamics Conference, 2013.

[10] Michael A. Park, William L. Kleb, William T. Jones, Joshua A. Krakos, Todd R. Michal, Adrien Loseille, Robert Haimes and John Dannenhoffer, "Geometry Modeling for Unstructured Mesh Adaptation", AIAA Paper 2019-2946, https://doi.org/10.2514/6.2019-2946

[11] Christopher M. Meckstroth, "Parameterized, Multi-fidelity Aircraft Geometry and Analysis for MDAO Studies using CAPS”, AIAA Paper 10.2514/6.2019-2230

[12] Carlier P., Debelmas C., Pilon J.C., Vélot-Leroux A., "Avant-projet d'un avion de transport commercial supersonique”, Aérospatiale-ENAC 1992

[13] Hoak D.E., Finck R.D., "USAF Stability and control DATCOM”, AFFDL, Wright Patterson AFB, Ohio (October 1960-Revised January 1975)

[14] Raymer D.P., “Aircaft design : a conceptual approach”, AIAA Education Series, 5th edition, Sep 2012.

[15] Steve Karman, Nick Wyman, "Automatic Unstructured Mesh Generation with Geometry Attribution”, AIAA Paper 10.2514/6.2019-1721

[16] “OpenCASCADE.” [Online]. Available: https://www.opencascade.com/.

[17] Palacios, F., Colonno, M. R., Aranake, A. C., Campos, A., Copeland, S. R., Economon, T. D., Lonkar, A. K., Lukaczyk, T. W., Taylor, T. W. R., and Alonso, J. J., "Stanford University Unstructured (SU2): An Open-source Integrated Computational, Environment for Multi-Physics Simulation and Design", 51st AIAA Aerospace Sciences Meeting, Grapevine, Texas

[18] E. S. Hendricks, J. S. Gray, « pyCycle: A Tool for Efficient Optimization of Gas Turbine Engine Cycles », Aerospace, vol. 6, nº 8, Art. nº 8, August 2019, doi: 10.3390/aerospace6080087.

[19] J. R. Stone, D. E. Groesbeck, C. L. Zola, “Conventional Profile Coaxial Jet Noise Prediction”, AIAA Journal, Vol. 21, No. 3, pp. 336-342, doi: 10.2514/3.8077

[20] Melissa B. Carter, Michael A. Park, "Near Field Summary and Analysis of the Third AIAA Sonic Boom Prediction Workshop Shock-Plume Interaction Case”, AIAA Paper AIAA 2021-0469

[21] Sriram K. Rallabhandi, Alexandra Loubeau, "Summary of Propagation Cases of the Third AIAA Sonic Boom Prediction Workshop”, AIAA Paper AIAA 2021-0229

[22] Whitham, G.B., "The Flow Pattern of a Supersonic Projectile,"Communications on pure andapplied mathematics, Vol. 5, No.3, pp. 301-348, 1952.

[23] Walkden, F., "The Shock Pattern of a Wing-Body Combination, far from the Flight Path”,Aeronautical quarterly, Vol. IX, pp. 164-194, 1958. 
[24] I. Salah El Din, M.-C. Le Pape, A. Minelli, R. Grenon, G. Carrier, «Impact Of Multipole Matching Resolution On Supersonic Aircraft Sonic Boom Assessment», Progress in Flight Physics5 (2013) 601-620

[25] A. D. Taylor, «The TrapsSonic Boom Program», NOAA TechnicalMemorandumERL ARL-87, July 1980 Air ResourcesLaboratories, SilverSpring, Maryland

[26] F. Coulouvrat, "Théorie géométrique non linéaire de la diffraction en zone d'ombre », C. R. Acad. Sci. Paris, 325, Série II b, 69-75, 1997 